\title{
Feasibility of noncontact piezoelectric detection of photoacoustic signals in tissue-mimicking phantoms
}

\author{
Roy G. M. Kolkman \\ University of Twente \\ MIRA Institute for Biomedical Technology and Technical \\ Medicine \\ BioMedical Photonic Imaging \\ P.O. Box 217 \\ 7500 AE Enschede, The Netherlands
}

\section{Erik Blomme \\ Tijl Cool}

Mattias Bilcke

Katholieke Hogeschool Zuid-West-Vlaanderen (KATHO)

Catholic University of Leuven Association

Lab NCU

Doorniksesteenweg 145, 8500 Kortrijk, Belgium

\section{Ton G. van Leeuwen}

University of Twente

MIRA Institute for Biomedical Technology and Technical Medicine

BioMedical Photonic Imaging

P.O. Box 217

7500 AE Enschede

The Netherlands and

University of Amsterdam

Academic Medical Center

Biomedical Engineering \& Physics

P.O. Box 22700

1100DE Amsterdam, The Netherlands

\author{
Wiendelt Steenbergen \\ University of Twente \\ MIRA Institute for Biomedical Technology and Technical \\ Medicine \\ BioMedical Photonic Imaging \\ P.O. Box 217 \\ 7500 AE Enschede, The Netherlands
}

Kees A. Grimbergen

University of Amsterdam

Academic Medical Center

Biomedical Engineering \& Physics

P.O. Box 22700

1100DE Amsterdam, The Netherlands and

Delft University of Technology

Faculty of Mechanical, Maritime and Materials Engineering (3mE)

Department of Biomechanical Engineering (BMechE)

Mekelweg 2

2628CD Delft, The Netherlands

Address all correspondence to: Roy G. M. Kolkman, University of Twente, MIRA Institute for Biomedical Technology and Technical Medicine, BioMedical Photonic Imaging, P.O. Box 217, Enschede, 7500AE, The Netherlands. Tel: 3153 489 2573; Fax: 3153489 1105; E-mail: r.g.m.kolkman@utwente.nl

\author{
Gerard J. den Heeten \\ University of Amsterdam \\ Academic Medical Center \\ Department of Radiology \\ P.O. Box 22700 \\ 1100 DE Amsterdam, The Netherlands \\ and \\ Dutch National Training and Reference Centre for Breast \\ Cancer Screening \\ P.O. Box 6873 \\ 6503 GJ Nijmegen, The Netherlands
}

\begin{abstract}
The feasibility of air-coupled ultrasound transducers to detect laser-induced ultrasound from artificial blood vessels embedded in an optically scattering phantom is demonstrated. These air-coupled transducers allow new applications in biomedical photoacoustic imaging where contact with tissue is not preferred. One promising application of such transducers is the addition of photoacoustic imaging to the regular x-ray mammographic screening procedure. (0) 2010 Society of Photo-Optical Instrumentation Engineers. [DOI: 10.1117/1.3491113]
\end{abstract}

Keywords: photoacoustic; optoacoustic; ultrasound; blood vessel; imaging; air-coupled ultrasound; air-coupled detection.

Paper 10219LRR received Apr. 23, 2010; revised manuscript received Aug. 22, 2010; accepted for publication Aug. 26, 2010; published online Sep. 23, 2010.

\section{Introduction}

Photoacoustic imaging is a novel imaging modality based on the use of laser-generated ultrasound by optically absorbing structures. Due to its ability to visualize increased hemoglobin concentrations, photoacoustic imaging is being explored as a noninvasive method to detect the vascularization of tumors. ${ }^{1-4}$ Photoacoustic mammography (PAM) $)^{5-7}$ is a new approach in the detection of breast cancer that is based on laser-induced ultrasound originating from invasive tumors containing more hemoglobin than in normal tissue due to extensive neovascularisation. ${ }^{8}$

Detection of laser-induced ultrasound is in general carried out by using piezoelectric transducers in combination with an ultrasonic coupling medium to avoid the large acoustic impedance mismatch between tissue and air. The need for this coupling medium makes application of photoacoustic imaging more complicated in cases where direct contact with the tissue has to be avoided, or in cases where one wants to combine photoacoustic imaging with other imaging modalities. A promising example where photoacoustic imaging can add additional information to existing imaging techniques is mammographic breast cancer screening.

Although mammographic breast cancer screening is an undisputed factor in the reduction of breast cancer mortality, the way to screen ${ }^{9}$ (starting age, screening interval) and the negative side effects like false positive test results are still causing

$1083-3668 / 2010 / 15(5) / 055011 / 4 / \$ 25.00$ @ 2010 SPIE 
debates in scientific society. ${ }^{10}$ The appraisal of these negative side effects can differ strongly between countries, where recall rates can vary by a factor 10 with no clear effects on detection. $^{11}$

Although considered very successful in screening, x-ray mammography is a far from perfect diagnostic modality. Even in a country like the Netherlands with extremely low recall rates (percentage of women called back for a diagnostic mammogram after the first $x$-ray screening), in every six recalls five are false positives, ${ }^{12,13}$ which cause a lot of stress and unnecessary anxiety. Recall is attributed to the inherent weakness of the x-ray contrast mechanism, the lack of contrast between normal fibroglandular and malignant tissue, which radiologists in the screening have to cope with. Since the purpose of screenings is to find early-stage tumors before they cause symptoms in a normal and healthy population, radiologists are looking for these small and often subtle mammographic signs. None of the false-positive mammographic abnormalities show any degree of neovascularisation like invasive breast cancer. ${ }^{8}$

On the other hand, PAM has potentially the ability to detect increased vascularizations, and therefore PAM can assist the radiologist in the detection of these tumors.

Since we know that even large tumors can remain hidden from x-ray mammography in dense breast tissue, an increased photoacoustic signal due to increased hemoglobin concentrations in absence of any mammographic abnormality might trigger the radiologist to have a second look or a follow-up examination.

Combining photoacoustic imaging with $\mathrm{x}$-ray screening of breast cancer will add additional information to this procedure. A combined x-ray and ultrasound system for therapy was developed by Novak et al., which shows that a combination of $\mathrm{x}$-ray and ultrasound is feasible. ${ }^{14}$ A disadvantage of current detectors used in photoacoustic imaging is that they need to be in contact with the tissue directly or via an ultrasonic coupling medium (water, or gel), which makes integration in a standard $\mathrm{x}$-ray screening procedure more complicated and more time consuming.

Attempts toward noncontact detection of laser-induced ultrasound that are reported in the literature were based on optical detection of tissue-surface displacement. ${ }^{15,16}$ However, these systems put requirements on the tissue surface as well as stability of the entire detection system. A solution to this can be found in the use of air-coupled ultrasound transducers used for noncontact material characterization ${ }^{17-19}$ and biomedical applications such as burn-depth estimation. ${ }^{20}$ We hypothesize that when these transducers can be used to detect photoacoustic pressure transients, they can be integrated in an x-ray mammography system to obtain additional information, without any interference with the mammographic procedure or the resulting image quality. The obtained information can be very useful in obvious mammographic abnormalities, but also and especially when an abnormal photoacoustic signal is received in an apparently normal mammogram, thereby theoretically improving the sensitivity and specificity of the mammographic screening test.

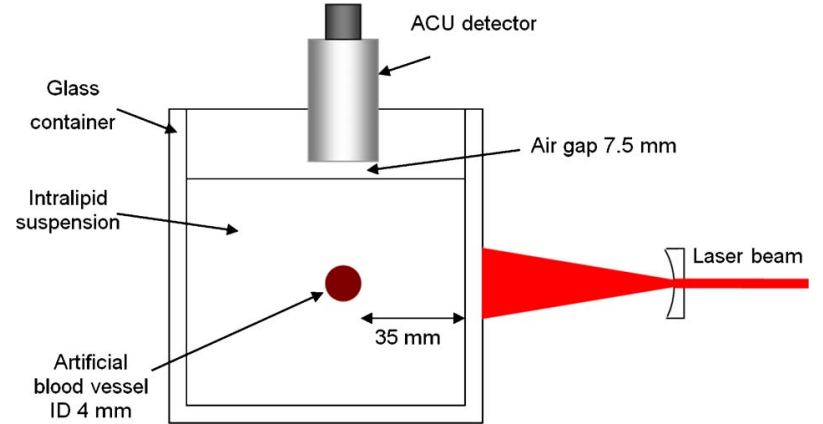

Fig. 1 Schematic drawing of the experimental setup: an artificial blood vessel with an internal diameter (ID) of $4 \mathrm{~mm}$ is placed in a glass container filled with an Intralipid suspension. The artificial blood vessel was illuminated through the wall of the glass container, and the generated photoacoustic signals were detected with an air-coupled ultrasound transducer placed approx. $7.5 \mathrm{~mm}$ above the Intralipid interface.

\section{Materials and Methods}

In this study the feasibility of using air-coupled ultrasound (ACU) transducers to detect photoacoustic signals has been studied in tissue-mimicking phantoms.

An artificial blood vessel made of a silicon rubber tube with an inner diameter of $4 \mathrm{~mm}$ was filled with human blood (anti-coagulated with EDTA). This artificial blood vessel was immersed in a 1\% Intralipid-20\% dilution with a reduced optical scattering coefficient of $0.14 \mathrm{~mm}^{-1}$ at a wavelength of $1064 \mathrm{~nm}^{21}$ and an optical absorption coefficient of $0.015 \mathrm{~mm}^{-1}$. The vessel was positioned in a glass container and illuminated through the wall of the container, while detection was carried out above the fluid surface. Laser light at a wavelength of $1064 \mathrm{~nm}$ was applied, as hemoglobin in blood has sufficient absorption at this wavelength to generate photoacoustic signals. A pulse energy of $200 \mathrm{~mJ} /$ pulse (pulseto-pulse stability better than $5 \%$ ) was used with a repetition rate of $10 \mathrm{~Hz}$ and a pulse duration of $10 \mathrm{~ns}$ (Quanta Ray DCR-3, Spectra Physics, Newport Corporation, Irvine, California). The laser light had to travel over a distance of $35 \mathrm{~mm}$ through the Intralipid suspension to reach the artificial blood vessel. The laser beam was expanded to illuminate an area of about $10 \mathrm{~cm}^{2}$, which resulted in an energy density at the interface of about $20 \mathrm{~mJ} / \mathrm{cm}^{2}$. A schematic drawing of the setup is shown in Fig. 1.

Laser-generated ultrasound originating from the artificial blood vessel and propagating through the Intralipid solution reaches the liquid/air interface. Due to the high acoustic impedance $(Z)$ mismatch between the solution ( $Z=1.6$ MRayl) and the air $(Z=0.0004$ MRayl), only a small fraction of acoustic energy $(-30 \mathrm{~dB})$ enters the air gap. This signal is detected by an unfocused air-coupled ultrasonic piezotransducer, which is supplied with a matching layer to reduce the impact of the high acoustic impedance mismatch between the transducer surface and the ambient air from $-43 \mathrm{~dB}$ to approximately $-33 \mathrm{~dB}$. Two types of transducers have been used: one with sensor elements from the Murata Piezotite series $(200 \mathrm{kHz})$, and one with Ferroperm (Kvistgaard, Denmark) Soft Piezoceramics and a polymer layer $(1 \mathrm{MHz}){ }^{19}$ The first type had a central frequency of $200 \mathrm{kHz},-6-\mathrm{dB}$ 

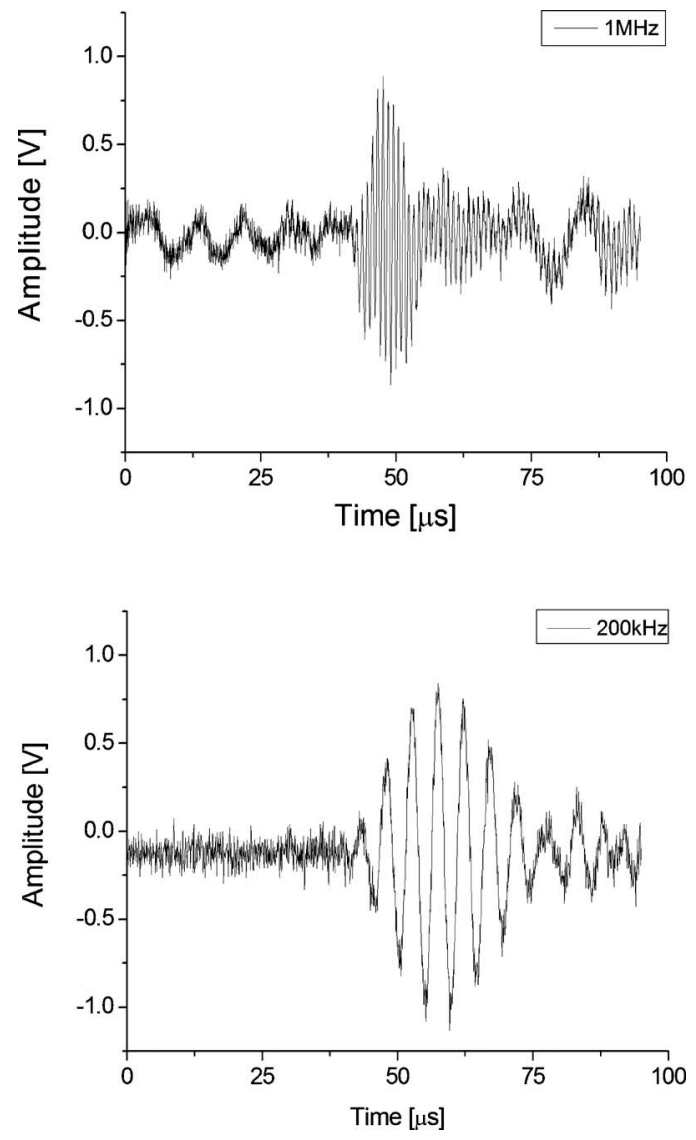

Fig. 2 Photoacoustic time traces from an artificial blood vessel with an inner diameter of $4 \mathrm{~mm}$, recorded with air-coupled ultrasound transducers with a center frequency of $200 \mathrm{kHz}$ and $1 \mathrm{MHz}$.

bandwidth of $50 \mathrm{kHz}$, and an active diameter of $9 \mathrm{~mm}$. The second type had a central frequency of $1 \mathrm{MHz},-6-\mathrm{dB}$ bandwidth of $90 \mathrm{kHz}$, and a $19-\mathrm{mm}$ active diameter. The transducer transformed the acoustic signal into an electric signal, and the latter is amplified by $60 \mathrm{~dB}$ using a homemade ultralow noise amplifier. The flatness of the amplifier response was better than $0.6 \mathrm{~dB}$ in the frequency band of interest $(200 \mathrm{kHz}$ to $1 \mathrm{MHz}$ ).

The distance between the ultrasonic receiver and the Intralipid solution was approximately $7.5 \mathrm{~mm}$.

The nonprocessed time traces from the air-coupled transducers were digitized by a dual-channel oscilloscope (100 MSamples/sec, TDS-220, Tektronix, Beaverton, Oregon) and were stored on a laptop computer.

\section{Results}

The photoacoustic time traces recorded by the noncontact transducers were averaged 512 times. The resulting averaged time traces are shown in Fig. 2. The photoacoustic signal appears as a burst, with an onset occurring at a time of $42 \mu \mathrm{s}$ after the laser pulse. This value is in agreement with the expected time of flight: to reach the air-coupled transducer, the photoacoustic signal had to travel through $30 \mathrm{~mm}$ of Intralipid suspension, which corresponds to a time delay of $20 \mu \mathrm{s}$ (the speed of sound in the $1 \%$-Intralipid- $20 \%$ suspension was measured to be $1495 \mathrm{~m} / \mathrm{s}$ at $25^{\circ} \mathrm{C}$ ), and an air gap with a dimension of $7.5 \mathrm{~mm}$, which corresponds to a time delay of $22 \mu \mathrm{s}$ (assuming a speed of sound in air of $345 \mathrm{~m} / \mathrm{s}$ at $25^{\circ} \mathrm{C}$ ).

The quasi-equal signal levels of the measurements by the noncontact transducers with a different center frequency have a purely coincidental nature. The explanation is found in the intrinsic broad but nonflat frequency spectrum of the photoacoustic signal and the differences in sensitivity, bandwidth, and active surface of the transducers.

The signal-to-noise ratio (SNR), defined as the ratio of the rms value of the burst to the rms value of the noise, was 16.8 in the case of the $1-\mathrm{MHz}$ transducer and 16.3 for the $200-\mathrm{kHz}$ transducer. Assuming a SNR improvement of $\sqrt{512}=22.6$, the original signal had a SNR of about 0.72 , and at least two averages of the photoacoustic time traces (i.e., two laser pulses) are required to achieve a SNR of 1 .

As can be observed in Fig. 2, the noise characteristics of the two sensors are different. This noise is mainly determined by the impedance characteristics of the piezoceramic in combination with the amplifier properties. Since the transducers used have a different design and operate in a different frequency domain, the noise characteristics are different.

\section{Discussion}

To our knowledge, air-coupled ultrasound transducers have so far not been used to detect photoacoustic signals in biomedical applications, which can be attributed to the large acoustic mismatch at the tissue-air interface of about 4 orders of magnitude. This acoustic mismatch allows only a fraction (about $0.1 \%$ ) of the ultrasound to be transmitted to the air, which is not only a problem in industrial nondestructive testing (NDT) techniques $^{18}$ but also in potential biomedical applications. Nevertheless, we have shown that the sensitivity of the aircoupled ultrasound transducers is sufficient to detect photoacoustic signals generated by an artificial blood vessel. These signals were detected at a distance of $7.5 \mathrm{~mm}$ above the phantom interface, which in practice allows for noncontact scanning over a tissue surface in case an image has to be obtained. Larger distances are possible; however, significant signal losses due to acoustic energy absorption in air and wave divergence should be taken into account. While wave divergence is determined by the complex morphologic nature of the unknown source, the acoustic energy absorption can well be estimated. Typically, a 1-MHz wave experiences an attenuation of $1.6 \mathrm{~dB}$ per $\mathrm{cm}$ in air. ${ }^{22}$

The attenuation expressed in decibels scales with about the frequency squared. This means that for the detection of higher frequencies, smaller air gaps have to be used. In this work, we have shown that signals with a frequency content up to $1 \mathrm{MHz}$ can be detected. This corresponds to photoacoustic sources with a diameter larger than $1.5 \mathrm{~mm},{ }^{23}$ which is already sufficiently small for mammographic breast cancer screening.

A drawback of the air-coupled transducers is the limitation on the frequency bandwidth, which determines the duration of the detected photoacoustic pressure burst. As is observed in Fig. 2, the duration of the detected ultrasound burst is about $12 \mu \mathrm{s}$ in the case of the $1-\mathrm{MHz}$ transducer and $39 \mu \mathrm{s}$ in case of $200-\mathrm{kHz}$ transducer, while in contact mode using a broadband transducer, a bipolar signal with a peak-to-peak time of 
$1.33 \mu$ s would have been detected. ${ }^{23}$ This long burst length limits the axial resolution of the photoacoustic imaging system in the case of the $200-\mathrm{kHz}$ transducer to $13 \mathrm{~mm}$ and in the case of the $1-\mathrm{MHz}$ transducer to $4 \mathrm{~mm}$. However, the latter might be improved by using signal processing algorithms such as deconvolution with the impulse response of the transducer. Furthermore, air-coupled transducers are subjects of permanent product improvement with bandwidth and sensitivity as main concerns. ${ }^{24}$

Since we demonstrated that air-coupled detection of photoacoustic signals from absorbers hidden in turbid media is feasible, this allows for integration of photoacoustic detection in the x-ray mammographic screening procedure without interfering with the standard screening procedure and image quality. During the time that the x-ray mammogram is being taken, the breast is illuminated with pulsed light and the aircoupled transducers can be used to detect the presence of malignant tissue by the presence of enlarged blood concentrations due to increased vascularization.

At this moment, the available air-coupled transducers are nonfocused, which makes obtaining an image complicated. However, combination of the signals of the various aircoupled transducers will enable a rough localization of the enlarged blood concentrations of T1 tumors (smaller than $2 \mathrm{~cm}$ in diameter). Being able to predict the presence of these suspicious regions in one of the four quadrants of the breast will add useful information to the mammographic screening procedure.

Other research groups are working on the development of air-coupled arrays. ${ }^{25,26}$ In the future, this might extend our proposed photoacoustic screening procedure to obtain a complete photoacoustic image of the breast.

In conclusion, we demonstrate the feasibility of aircoupled ultrasound transducers to detect photoacoustic signals in biomedical applications, which opens new possibilities of (combined) imaging with other modalities such as X-ray mammography. Besides application in photoacoustic mammographic screening, air-coupled detection of photoacoustic signals allows for a variety of applications, especially in situations where contact with the tissue via ultrasound matching gel is not desired, such as measurements on burn wounds.

\section{References}

1. R. I. Siphanto, K. K. Thumma, R. G. M. Kolkman, T. G. van Leeuwen, F. F. M. de Mul, J. W. van Neck, L. N. A. van Adrichem, and W. Steenbergen, "Serial noninvasive photoacoustic imaging of neovascularization in tumour angiogenesis," Opt. Express 13, 89-95 (2005)

2. G. Ku, X. Wang, X. Xie, G. Stoica, and L. V. Wang, "Imaging of tumor angiogenesis in rat brains in vivo by photoacoustic tomography," Appl. Opt. 44, 770-775 (2005).

3. Y. Lao, Y. D. Xing, D. S. Yang, and L. Xiang, "Noninvasive photoacoustic imaging of the developing vasculature during early tumor growth," Phys. Med. Biol. 53, 4203-4212 (2008).

4. H. F. Zhang, K. Maslov, G. Stoica, and L. V. Wang, "Functional photoacoustic microscopy for high-resolution and noninvasive in vivo imaging," Nat. Biotechnol. 24, 848-851 (2006).

5. S. Manohar, S. E. Vaartjes, J. C. G. van Hespen, J. M. Klaase, F. M. van den Engh, W. Steenbergen, and T. G. van Leeuwen, "Initial results of in vivo non-invasive cancer imaging in the human breast using near-infrared photoacoustics," Opt. Express 15, 12277-12285 (2007).

6. T. Khamapirad, P. M. Henrichs, K. Mehta, T. G. Miller, A. T. Yee, and A. A. Oraevsky, "Diagnostic imaging of breast cancer with LOIS: clinical feasibility," Proc. SPIE 5697, 35-44 (2005).

7. R. A. Kruger, K. D. Miller, H. E. Reynolds, W. L. Kiser Jr., D. R. Reinecke, and G. A. Kruger, "Breast cancer in vivo: contrast enhancement with thermoacoustic CT at $434 \mathrm{MHz}$-feasibility study," Radiology 216, 279-283 (2000).

8. P. Carmeliet and R. K. Jain, "Angiogenesis in cancer and other diseases," Nature 407, 249-257 (2000).

9. "Screening for breast cancer: U.S. Preventive Services Task Force recommendation statement," Ann. Intern Med. 151(10), 716-736 (2009).

10. P. C. Gøtzsche and M. Nielsen "Screening for breast cancer with mammography," Cochrane Database System. Rev. 4, CD001877 (2009).

11. B. C. Yankaskas, C. N. Klabunde, R. Ancelle-Park, G. Renner, H Wang, J. Fracheboud, G. Pou, and J. L. Bulliard, International Breast Cancer Screening Network, "International comparison of performance measures for screening mammography: can it be done?" $J$. Med. Screen 11(4), 187-93 (2004).

12. J. H. Groenewoud, J. D. Otten, J. Fracheboud, G. Draisma, B. M. van Ineveld, R. Holland, A. L. Verbeek, and H. J. de Koning, "Costeffectiveness of different reading and referral strategies in mammography screening in the Netherlands," Breast Cancer Res. Treat 102, 211-218 (2007)

13. NETB (National Evaluation Team for Breast cancer screening in the Netherlands), LETB XII (2009) National Evaluation of Breast Cancer Screening in the Netherlands, Vol. 12, Department of Public Health, Eraxmus MC, Rotterdam (2009).

14. P. Novák, E. G. Moros, W. L. Straube, and R. J. Myerson, "SURLAS: a new clinical grade ultrasound system for sequential or concomitant thermoradiotherapy of superficial tumors: applicator description," Med. Phys. 32(1), 230-240 (2005).

15. B. P. Payne, V. Venugopalan, B. B. Mikić, and N. S. Nishioka, "Optoacoustic tomography using time-resolved interferometric detection of surface displacement," J. Biomed. Opt. 8(2), 273-280 (2003).

16. S. L. Jacques, P. E. Andersen, S. G. Hanson, and L. R. Lindvold, "Noncontact detection of laser-induced acoustic waves from buried absorbing objects using a dual-beam common-path interferometer,' Proc. SPIE 3254, 307-318 (1998).

17. M. C. Bhardwaj, "Non-destructive evaluation," in Encyclopedia of Smart Materials, John Wiley and Sons, New York, pp. 690-714 (2002)

18. E. Blomme, D. Bulcaen, and F. Declercq, "Air-coupled ultrasonic NDE: experiments in the frequency range $750 \mathrm{kHz}-2 \mathrm{MHz}, " N D T$ \& E Int. 35, 417-426 (2002).

19. T. E. Gómez, "Acoustic impedance matching of piezoelectric transducers to the air," IEEE Trans. Ultrason. Ferroelectr. Freq. Control 51, 624-633 (2004).

20. J. P. Jones, D. Lee, M. Bhardwaj, V. VanderKam, and B. Achauer, "Non-contact ultrasonic imaging for the evaluation of burn depth and other biomedical applications," Acoust. Imaging 23, 89-93 (1997).

21. H. J. van Staveren, C. J. M. Moes, J. van Marle, M. J. C. van Gemert, and S. Prahl, "Light scattering in Intralipid-10\% in the wavelength range of 400-1100 nm," Appl. Opt. 30, 4507-4514 (1991).

22. "Ultrasonic testing," Nondestructive Testing Handbook, A. S. Birks R. E. Green, and P. McIntire, Eds., pp. 320-325, ASNT, Columbus, $\mathrm{OH}$ (1991).

23. R. G. M. Kolkman, J. H. G. M. Klaessens, E. Hondebrink, J. C. W. Hopman, F. F. M. de Mul, W. Steenbergen, J. M. Thijssen, and T. G. van Leeuwen, "Photoacoustic determination of blood vessel diameter," Phys. Med. Biol. 49, 4745-4756 (2004).

24. J. Kunkle, R. Vun, T. Eischeid, M. Langron, N. Bhardwaj, and M Bhardwaj, "Phenomenal advancements in transducers and piezoelectric composites for non-contact ultrasound and other applications," Proc. European Conf. Non-Destructive Testing, p. Fr.2.3.1, Berlin, Germany (2006).

25. A. I. Korobov, M. Y. Izosimova, and S. A. Toschov, "Development of ultrasound focusing array for air-coupled ultrasound generation," Phys. Procedia 3, 201-207 (2010).

26. F. Blum, J. Jarzynski, and L. J. Jacobs, "A focused two-dimensional air-coupled ultrasonic array for non-contact generation," $N D T \& E$ Int. 38, 634-642 (2005) 